\title{
Impact of Electrode Area, Contact Impedance and Boundary Shape on EIT Images
}

\author{
Alistair Boyle and Andy Adler \\ Systems and Computer Engineering, Carleton University, Ottawa, ON, Canada \\ E-mail: boyle@sce.carleton.ca, adler@sce.carleton.ca
}

\begin{abstract}
Electrical Impedance Tomography (EIT) measures the conductivity distribution within an object based on the current applied and voltage measured at surface electrodes. Thus, EIT images are sensitive to electrode properties (i.e. contact impedance, electrode area, and boundary shape under the electrode). While some of these electrode properties have been investigated individually, this paper investigates these properties and their interaction using Finite Element Method (FEM) simulations and the Complete Electrode Model (CEM). The effect of conformal deformations on image reconstruction when using the CEM was of specific interest. Observed artifacts were quantified using a measure that compared an ideal image to the reconstructed image, in this case a nonoise reconstruction that isolated the electrodes' effects. For electrode contact impedance and electrode area, uniform reductions to all electrodes resulted in ringing artifacts in the reconstructed images when the CEM was used, while parameter variations that were not correlated amongst electrodes resulted in artifacts distributed throughout the image. When the boundary shape changed under the electrode, as with non-symmetric conformal deformations, using the CEM resulted in structured distortions within the reconstructed image. Mean electrode contact impedance increases, independent of inter-electrode variation, did not result in artifacts in the reconstructed image.
\end{abstract}

Keywords: Electrical Impedance Tomography, Finite Element Method, Complete Electrode Model, conformal deformation Submitted to: Physiol. Meas.

\section{Introduction}

Electrical Impedance Tomography (EIT) applies current and measures the resulting voltage on the surface of a target. In biomedical applications, this current is applied, and voltage is measured, through electrodes attached to the body. Models are used to represent these electrode connections in the reconstruction of the conductivity image, tying circuit models to Finite Element Method (FEM) simulations. Changes in the contact impedance or boundary shape relative to the electrode's surface area can introduce artifacts in the reconstructed image. The quantity and quality of these artifacts is dependant upon the electrode model and the properties assigned to that model.

The electrode models were originally formulated in the context of mathematical proofs of solution existence and uniqueness for EIT (Calderón 2006, Nachman 1996). The Complete Electrode Model (CEM) allows a complex impedance for each electrode 
Impact of Electrode Area, Contact Impedance and Boundary Shape on EIT Images 2

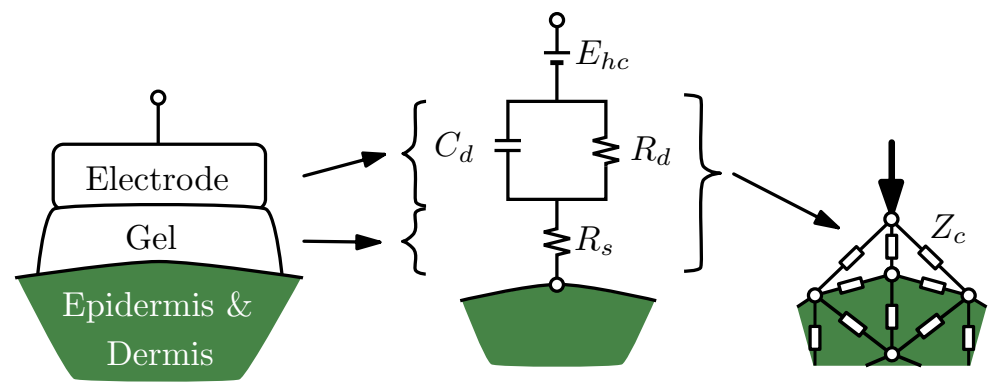

Figure 1: Generalized electrode model, adapted from (Webster 1998, McAdams et al. 1996), $E_{h c}$ electrode half-cell potential, $R_{d} C_{d}$ electrode impedance, $R_{s}$ gel related impedance, $R_{s}+\left(R_{d} \|-\mathrm{i} / \omega C_{d}\right)=Z_{c}$ contact impedance. Note that if a quasistatic reconstruction is assumed ( $\omega \simeq 0$ ), the parallel capacitance may be ignored, leaving only the resistive component of the contact impedance.

that models the metal electrode, conductive gel and chemical interaction at the skinelectrode interface (Cheng et al. 1989, Somersalo et al. 1992). The FEM is used in the numerical solution of EIT images. The simplest electrode model to implement in the FEM is the Point Electrode Model (PEM) which applies current and measures voltage at single nodes on the boundary and requires no further equations to implement. The PEM does not consider the geometry or contact impedance of an electrode. To reconstruct accurate images from in vivo data, an accurate electrode model is frequently required, and thus, the CEM is generally preferred (Cheng et al. 1989). (Figure 1)

For absolute image reconstruction, there can be a significant requirement for correct specification of electrode impedance (Kolehmainen et al. 1997). However, for difference imaging, electrode impedance is not as critical because it is commonly assumed in the reconstruction process that the contact impedance remains constant and that all measurement changes are due to internal conductivity changes. This may not be an accurate assumption; over time the contact impedance of electrodes changes largely due to sweat on the skin and drying of the electrode gel. Changes will be minimized by difference imaging when done over short intervals relative to the change in contact impedance. Experience has shown that, at longer intervals, factors such as electronics drift and electrode contact changes result in poor quality reconstructions.

Electrode contact impedance is commonly defined in units of impedance and area $\left(\Omega \cdot \mathrm{m}\right.$ in $2 \mathrm{D}, \Omega \cdot \mathrm{m}^{2}$ in $\left.3 \mathrm{D}\right)$. For a CEM spanning multiple edges on the FEM mesh boundary, there must be a distribution of the contact impedance amongst those edges. One method of distributing the contact impedance is to use a linear expansion function that assigns the contact impedance based on the length between nodes in two dimensions or area of a boundary element in three dimensions as in (Polydorides \& Lionheart 2002). Because contact impedance is typically specified in units that include geometry, the location of the FEM nodes for the electrode must accurately reflect the total area of the electrode to achieve the correct overall electrode impedance. In vivo, the electrodes are generally fabric or plastic backed and do not stretch as a deformation such as a dilation occurs. If the area of the electrodes would have changed to match the deformation but for the backing of the electrode, anomalies will be introduced into 
the reconstructed image because of the change in boundary definition. If the shape of the boundary changes beneath the electrode, the direction of the current vector can change relative to the region being imaged, again resulting in anomalies in the reconstructed image.

Undesirable localized anomalies in the reconstructed image are referred to as artifacts. Image artifacts can be quantified using a measure such as the Artifact Amplitude Measure (AAM), the sum of the squared normal error of an image's conductivity elements. Artifacts can have different appearances not captured by a single quantitative measure such as the AAM. Practitioners of EIT would be most familiar with a generalized noise in the reconstructed image which grows in intensity as the generating factors, such as measurement noise, increase. Other artifacts with a more symmetric geometry can occur, such as "ringing" (similar to an unbalanced transmission line) that results in circular waves in the image. The most subtle artifacts captured by a measure such as the AAM are those that result from a distortion within the reconstructed image. With these subtle differences, a superficial observation does not necessarily reveal that a distortion of the reconstruction has occurred and that, therefore, the geometric shape of a reconstructed target may be flawed.

When conformal movements of the boundary, such as dilation, occur there is no change in the reconstructed image (i.e. no artifacts) when using a PEM (Boyle et al. 2008). It is possible to use analytic solutions for boundary deformation when no electrode model is used, but currently, there is no analytic expression to capture the anisotropic conductivity changes caused by deformations when electrode models are used. We opted to use simulations to investigate these effects as a result of the lack of an analytic framework.

We investigated the relationship between electrode contact impedance, electrode area, and boundary shape under the electrode upon two-dimensional difference EIT image reconstruction quality using the CEM. The effect of two-dimensional conformal deformations on the reconstruction was of specific interest.

\section{Method}

The effect of electrode contact impedance and electrode area changes were investigated by independently manipulating the contact impedance, area, and boundary shape, while their effect on the reconstructed image was observed. Simulations were performed, using Electrical Impedance and Diffuse Optics Reconstruction Software (EIDORS) with NetGen (Adler \& Lionheart 2006, Schöberl 1997), based on a twodimensional approximation of a circular tank model with homogeneous conductivity (33439 elements, $1 \mathrm{~m}$ dia. circular domain, 16 CEM $0.2 \mathrm{~m}$ electrodes, background conductivity of $1 \mathrm{~S} / \mathrm{m}$ ) containing circular and rectangular targets (conductivity 2 $\mathrm{S} / \mathrm{m}$ ). The measurements were reconstructed using a course mesh (7207 elements, Tikhonov single step inverse solver $\lambda=1 \mathrm{e}-5$, Tikhonov image prior). The PEM was used for comparison where appropriate. Image artifacts were quantified using the AAM (1), the sum of the squared normal error of an image's conductivity elements $\mathbf{c}_{1}$, where the error was relative to some ideal conductivity reconstruction $\mathbf{c}_{0}$.

$$
\mathrm{AAM}_{n}=\sum\left[\frac{\mathbf{c}_{1}-\mathbf{c}_{0}}{\left|\mathbf{c}_{0}\right|_{\max }}\right]^{2}
$$

To isolate the effect of electrode properties from the effects of regularization on the solution, the ideal conductivity $\mathbf{c}_{0}$ was taken as the regularized solution of a 
reconstruction with no measurement noise or electrode variation. This choice of ideal reconstruction $\mathbf{c}_{0}$ is not possible in vivo where the precise expected solution is unknown.

As a baseline for comparison, a reconstruction with no electrode or measurement noise and a similar reconstruction with only measurement noise (50dB SNR difference measurements) were simulated.

Simulations of the effect of uncorrelated electrode impedance changes, as might be found with in vivo electrodes over time, were compared to the initial reconstructions. Electrode contact impedance $z_{c}[\Omega \cdot \mathrm{m}]$ was drawn from an exponential Gaussian distribution

$$
z_{c}=10^{\mathcal{N}}\left(\mu, \sigma^{2}\right)
$$

where $\mathcal{N}$ is a Gaussian distribution with a given mean $\mu$ and variance $\sigma^{2}$.

Contact impedance was specified in units that included geometry $(\Omega \cdot \mathrm{m})$. To manipulate electrode area independent of the total electrode impedance, the location of the FEM boundary nodes under the electrode had to reflect an appropriate area. When the total electrode impedance was held constant, by correcting contact impedance for any electrode area change, arbitrary deformations resulted in artifacts specific to the deformation. Changing the electrode properties simultaneously resulted in a compounding effect. Conformal deformations were used to isolate these two effects because conformal deformations change the forward problem geometry but do not result in changes in the reconstructed image when using the PEM (Boyle et al. 2008). Conformal deformations are those deformations that satisfy the Cauchy-Riemann equations

$$
\begin{aligned}
& \frac{\partial X_{1}}{\partial x_{1}}-\frac{\partial X_{2}}{\partial x_{2}}=0 \\
& \frac{\partial X_{1}}{\partial x_{2}}+\frac{\partial X_{2}}{\partial x_{1}}=0
\end{aligned}
$$

where $X$ is a vector field and $x$ is a deformation such that $X \mapsto x+X$ (Marsden $\&$ Hoffman 1998). The broader class of conformal deformations, which includes the isometric deformations, can be considered a localized isometric transformation at the infinitesimal scale. Isometric deformations (global reflection, rotation, or translation) are applied to the FEM mesh without altering the solution because the frame of reference, and thus the electrodes, are altered in an identical manner.

The behaviour of the PEM and CEM were explored under two types of conformal deformation: a $10 \%$ dilation, and a more complex deformation

$$
z=x+\mathrm{i} y ; \quad z \rightarrow z+0.4 z^{2}
$$

The forward model, before and after the conformal deformation of (5) was applied, is shown in Figure 2 using a CEM. The first set of measurements was simulated on the undeformed model with homogeneous conductivity, and the second set of measurements was simulated on the conformally deformed domain.

\section{Results}

Electrode contact impedance, area, and the boundary shape under the electrode were each separately manipulated, and their effect on the reconstructed image was observed. A conductivity image reconstructed on a coarse mesh with no measurement 


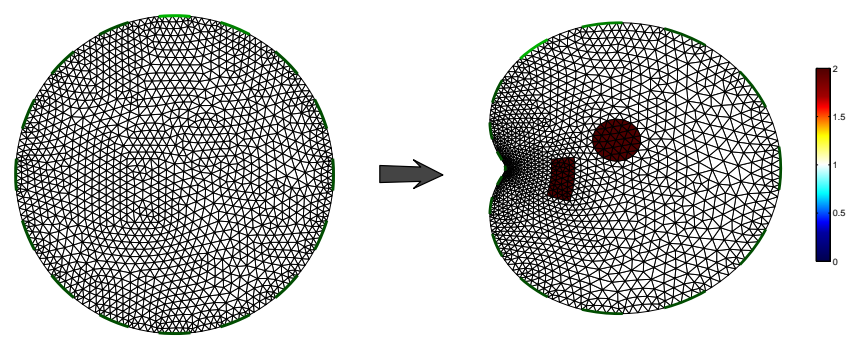

Figure 2: Complex conformal deformation: $z=x+\mathrm{i} y ; z \rightarrow z+0.4 z^{2}$. Forward model, dense mesh, CEM.

noise (Figure 3(a)) was used as the baseline for calculation of the AAM. With no measurement noise, the reconstructed image exhibited some low level variation in regions where the conductivity change should have been zero. These changes are attributable to the ill-conditioned nature of the problem and the choice of regularization scheme. Adding noise to the measurements (50dB SNR), modelled as Additive Gaussian White Noise (AGWN) (Figure 3(b)), increased the amplitude of the artifacts already present in the no-noise reconstruction but in general, did not cause artifacts to appear near the boundary.

To compare the effect of contact impedance change and electrode area change, electrode area (Figure 3(c)) and contact impedance (Figure 3(d)) were uniformly reduced to achieve similar AAM. Both area and contact impedance changes exhibited similar artifacts, ringing near the boundary. With electrode area reduced to $1 / 4$ $\left(A A M_{n}=5.5\right)$ and contact impedance reduced to $1 / 16\left(A A M_{n}=7.0\right)$ of their original values, artifacts of similar magnitude and location resulted.

Simulations were then performed over a range of electrode contact impedances drawn from the exponential normal distribution $(2)\left(\sigma^{2}=|\mu|\right)$. Both the quantity and magnitude of the artifacts increased with decreasing mean and corresponding increase in variance, as expected (Figures 3(e), 3(f), and 3(g)).

This effect on AAM was charted in Figure 4(a), where the parameters of the probability distribution (2) from which contact impedance was drawn were set with mean and variance equal $\left(\sigma^{2}=|\mu|\right)$, and the mean was varied over six orders of magnitude with 100 simulations at each mean value to establish error bars on the plot. It was striking that the resulting plot of AAM was not symmetric about the central, zero mean, and zero variance values. Decreases in the mean resulted in an increase in AAM, while increasing the mean resulted in relatively small changes to AAM. These results indicate that the mean of the contact impedance is a much more significant factor than variance in the generation of artifacts and the resulting AAM. To show solely the effect of mean contact impedance changes, a follow up experiment in which the variance was held constant at $\sigma^{2}=3$ and only the mean value changed was plotted (Figure 4(b)). The plot showed that the AAM changed significantly as a function of mean contact impedance.

Finally, the area of the electrode and the boundary shape under the electrode were varied while the overall contact impedance per-electrode remained constant. (Table 1) The performance of the two types of electrode model (PEM and CEM) were compared using the AAM. For each electrode model, two types of conformal deformation 


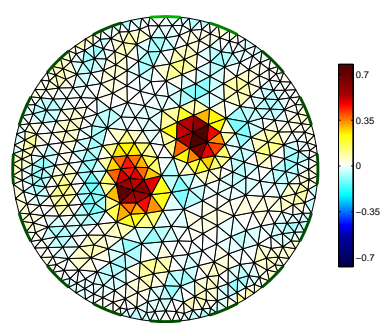

(a) no-noise

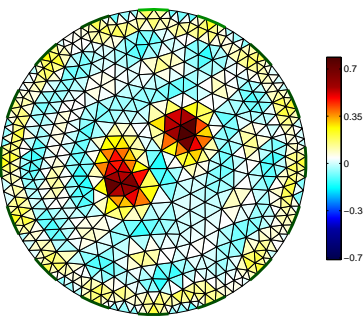

(d) contact impedance $\mu=$ $-1.2, \sigma^{2}=0$ (symmetric), reduced to $1 / 16, A A M_{n}=7.0$

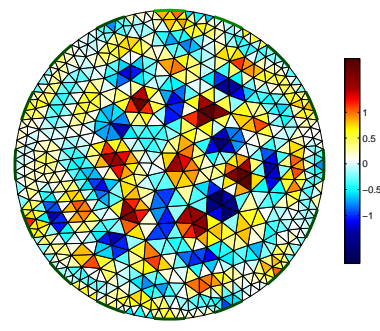

(g) contact impedance $\mu=$ $-1.5, \sigma^{2}=1.5, A A M_{n}=70.8$

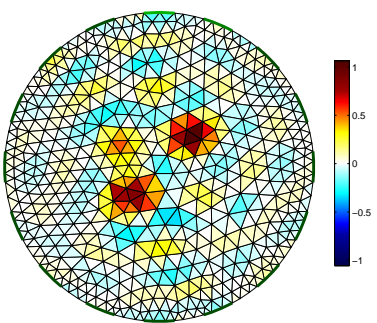

(b) measurement noise, 50dB SNR

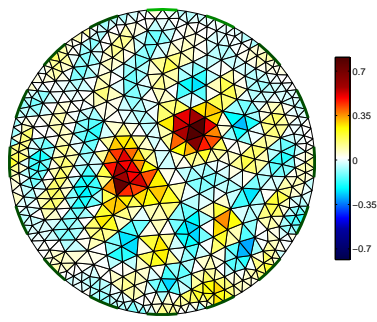

(e) contact impedance $\mu=$ $-0.25, \sigma^{2}=0.25, A A M_{n}=$ 8.1

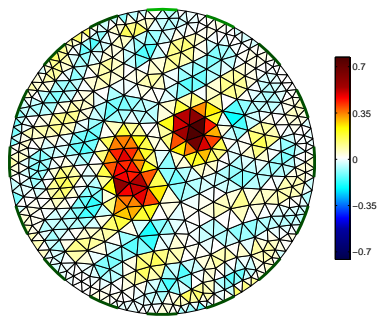

(h) electrode area (deformed targets), complex conformal deformation with flexible electrodes

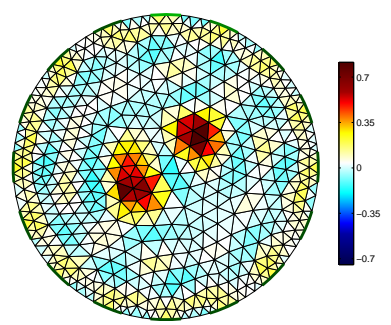

(c) electrode area (symmetric), reduced to $1 / 4, A A M_{n}=$ 5.5

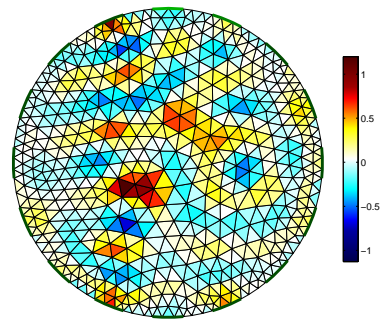

(f) contact impedance $\mu=$ $-0.75, \sigma^{2}=0.75, A A M_{n}=$ 26.2

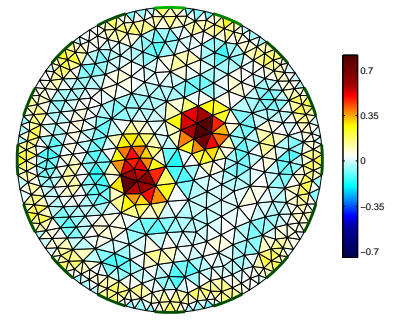

(i) electrode area (ringing around the boundary), $10 \%$ dilation, fixed area electrodes

Figure 3: Difference images of two targets with three types of artifacts exhibited due to the indicated "noise" source: ringing near the boundary $(\mathrm{c}, \mathrm{d}, \mathrm{i})$, distorted targets (h), and random localized anomalies (b, e, f, g), when compared to the no-noise reconstruction (a).

were applied: a $10 \%$ dilation and the more complicated conformal deformation (5). (Figure 2) These deformations are labelled "dilation" and "complex" respectively in the second column of Table 1. For the dilation deformation, the electrode area deformed with the boundary or the electrode area was held constant while the deformation of the rest of the domain occurred (Table 1, column 3, "matching" and "fixed", respectively). For the "complex" deformation, the AAM was only measured 
for electrodes deformed to match the boundary since holding the electrode shape constant through the deformation would result in a non-conformal deformation along the boundary and additional non-electrode related artifacts would occur as a result.

The simulations (reflected in Table 1) showed that the PEM was not affected by conformal changes, whether the electrode model fixed the area or changed to match the boundary. For the CEM, changes that were symmetric (e.g. dilation) did not result in significant artifacts if the electrode deformed with the boundary change. When the area of the CEM was fixed and a dilation occurred, "ringing" artifacts were observed. (Figure 3(i)) When the CEM was deformed in a complex conformal manner, deformation of the reconstructed image was observed as the source of the increased AAM. (Figure 3(h))

Table 1: Electrode Model Behaviour under Deformation

\begin{tabular}{ccccl}
\hline & \multicolumn{2}{c}{ Deformation } & & \\
\cline { 2 - 3 } Model & Domain & Electrode & $\mathrm{AAM}_{n}$ & Comment \\
\hline PEM & dilation & matching & 0 & \\
& complex & & 0.0807 & \\
\multirow{2}{*}{ CEM } & dilation & fixed & 0 & \\
& dilation & matching & 0.0010 & \\
& complex & & 2.013 & artifacts (deformed, Figure 3(h)) \\
& dilation & fixed & 5.5 & artifacts (ringing, Figure 3(i)) \\
\hline
\end{tabular}

\section{Discussion}

This work investigates the effect of errors in electrode contact impedance, electrode area, and boundary shape under the electrode in two-dimensional difference EIT reconstructions. The results show that using the CEM produces artifacts when conformal deformations are applied.

The results obtained for contact impedance variation simulations generally agree with previously published results (Boone \& Holder 1996) which indicated that as little as $20 \%$ variation in contact impedance can result in an image that has artifacts significant enough to render the image "almost meaningless." If the threshold for an acceptable level of artifacts is set at an AAM of 20, the simulation models show that a drop in average contact impedance amongst electrodes of $53 \%$ (variance 53\%) is enough to seriously affect the reconstruction quality. On the other hand, increases in contact impedance never reach unacceptable levels when the contact impedance variance amongst electrodes is within three orders of magnitude. In fact, the artifact levels due to contact impedance drop to nearly zero when the mean contact impedance increases by more than 1.5 orders of magnitude.

Typically in simulation, artifacts in difference EIT image reconstructions related to electrode model parameters do not arise because a common electrode model is used in the forward problem and its inverse solution. The electrode contact impedance, electrode area, and boundary shape under the electrode are assumed constant throughout and therefore, cancel in difference imaging. In vivo changes in these electrode properties do occur to some degree, and simulations that do not apply appropriate variation to the contact impedances are likely to get optimistic results. 
Impact of Electrode Area, Contact Impedance and Boundary Shape on EIT Images 8

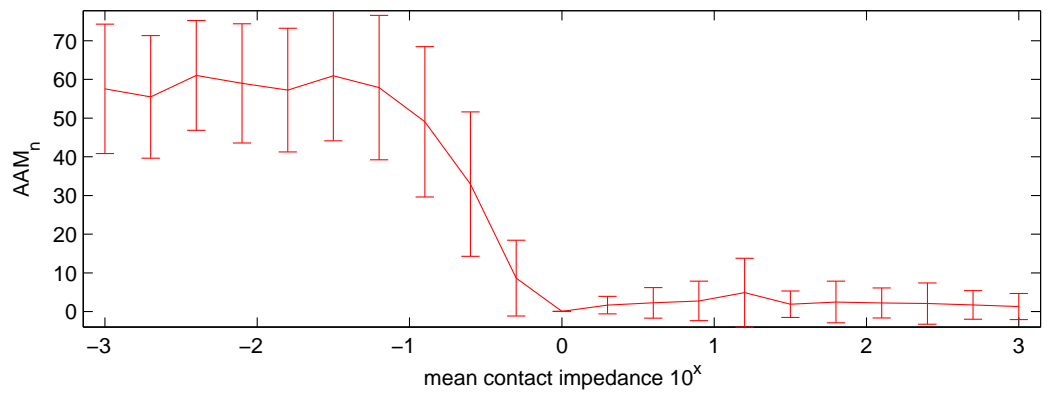

(a) $\sigma^{2}=|\mu|$

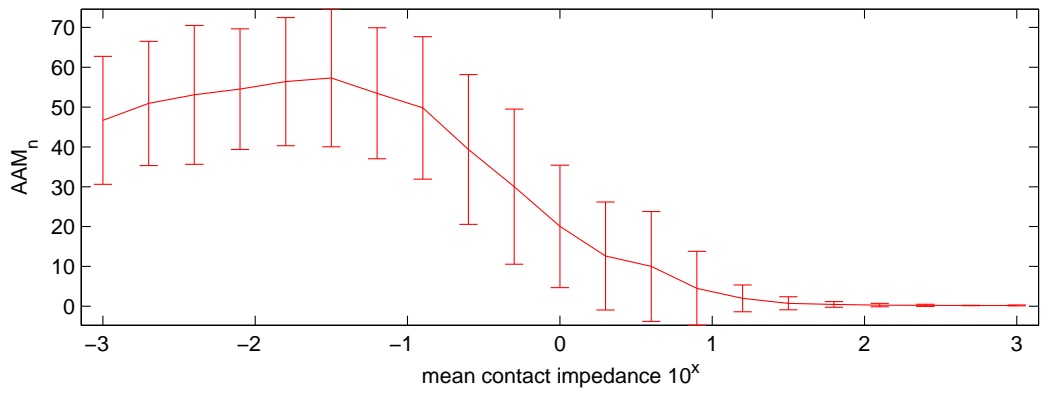

(b) $\sigma^{2}=3$

Figure 4: Mean contact impedance versus artifact amplitude: contact impedance drawn from $z_{c}=10^{\mathcal{N}}\left(\mu, \sigma^{2}\right)$ where mean was varied over 6 orders of magnitude $-3 \leq \mu \leq 3$ and (a) variance was matched to mean $\sigma^{2}=|\mu|$, (b) variance was fixed $\sigma^{2}=3$. Artifact amplitude measured with $A A M_{n}$, as the squared error versus a no-noise reconstruction normalized to the maximum no-noise conductivity.

When contact impedance increases, the reconstructions are largely artifact free. This behaviour is explained by considering the electrode model as a resistor network attached to the FEM, itself a low impedance resistor network. Large contact impedances mean that the voltage measurements at the electrode are approximately the average of the boundary node voltages connected to the electrode model; however, a small electrode contact impedance results in a voltage measurement at the electrode that is highly dependent on the surrounding FEM mesh's conductivity. In this environment, voltage measurements through small electrode contact impedances will be heavily affected by conductivity artifacts reconstructed near the boundary and are more likely to introduce these artifacts in the inverse problem.

In general biomedical and industrial applications, achieving a minimal contact impedance is desirable to maximize measurement sensitivity. The EIT simulations described in this study show that, with contact impedance variability, reconstruction artifacts can be a significant factor in image quality as contact impedance is reduced. This raises the possibility of attempting to reconstruct the electrode contact impedance as part of the inverse problem. In fact, some reconstructions of the CEM contact impedance have been successful under homogeneous conductivity conditions. These reconstructions included the CEM contact impedance as part of the inverse conductivity problem but required a uniform conductivity throughout 
Impact of Electrode Area, Contact Impedance and Boundary Shape on EIT Images 9

the interior region to maintain the uniqueness of the EIT conductivity solution (Vilhunen et al. 2002, Heikkinen et al. 2002, Hua et al. 1993). Increasing the electrode contact impedance or simultaneously reconstructing the contact impedance and interior conductivity through some form of regularization may mitigate the effects of contact impedance variation.

This study has shown that, for two-dimensional difference EIT conductivity reconstructions, electrode contact impedance, electrode area, and boundary shape under the electrode can be a significant source of artifacts when using the CEM.

\section{Acknowledgments}

This work was supported by the Natural Sciences and Engineering Research Council of Canada (NSERC).

\section{References}

Adler A \& Lionheart W R B 2006 Physiol. Meas. 27(5), S25-S42.

Boone K G \& Holder D S 1996 Medical and Biological Engineering and Computing 34(5), 351-354.

Boyle A, Lionheart W R B \& Adler A 2008 in '9th Conf. Electrical Impedance Tomography' Dartmouth College, Hannover, NH, USA.

Calderón A P 2006 Computational \& Applied Mathematics 25, 133-138. (reprint, orig. 1980).

Cheng K S, Isaacson D, Newell J C \& Gisser D G 1989 IEEE Trans. Biomed. Eng. 36(9), 918-924.

Heikkinen L M, Vilhunen T, West R M \& Vauhkonen M 2002 Meas. Sci. and Tech. 13(12), 1855-1861.

Hua P, Woo E J, Webster J G \& Tompkins W J 1993 IEEE Trans. Biomed. Eng. 40(4), 335-343.

Kolehmainen V, Vauhkonen M, Karjalainen P A \& Kaipio J P 1997 Physiol. Meas. 18, 289-303.

Marsden J \& Hoffman M 1998 Basic Complex Analysis W.H. Freeman \& Company.

McAdams E T, Jossinet J, Lackermeier A \& Risacher F 1996 Medical and Biological Engineering and Computing 34(6), 397-408.

Nachman A I 1996 The Annals of Mathematics 143(1), 71-96.

Polydorides N \& Lionheart W R B 2002 Meas. Sci. and Tech. 13(12), 1871-1883.

Schöberl J 1997 Computing and visualization in science 1(1), 41-52.

Somersalo E, Cheney M \& Isaacson D 1992 SIAM J. Appl. Math. 52(4), 1023-1040.

Vilhunen T, Kaipio J P, Vauhkonen P J, Savolainen T \& Vauhkonen M 2002 Meas. Sci. and Tech. 13(12), 1848-1854.

Webster J G, ed. 1998 Medical Instrumentation: Application and Design third edn John Wiley \& Sons New York. 